\title{
SENSIBILIDAD INTERCULTURAL DE LOS JÓVENES BILINGÜES VASCOS: ALGUNOS ASPECTOS A TRABAJAR EN EL SISTEMA EDUCATIVO VASCO
}

\author{
Naia Eguskiza \\ Juan Abasolo \\ Aintzane Etxebarria \\ Aitor Iglesias \\ Universidad del País Vasco
}

RESUMEN: El objetivo principal de esta investigación empírica es identificar fortalezas y aspectos a trabajar para atender y cuidar la diversidad en las aulas desde el punto de vista de la sensibilización intercultural. Para ello, tras realizar una revisión del marco legal en cuanto a los programas de atención a la diversidad, se ha recogido información mediante la herramienta Intercultural Sensitivity Scale (Escala de Sensibilidad Intercultural) de 410 estudiantes (223 alumnas y 187 alumnos) de la Comunidad Autónoma del País Vasco, junto con información descriptiva respecto a género, curso y lengua(s) materna(s). Los resultados del análisis de los datos indican que: la lengua materna es una variable a tener en cuenta, ya que es decisiva junto con el género o el curso de escolarización del alumnado. Además, se han identificado principalmente dos puntos importantes a trabajar en las aulas de la CAPV: la frustración y la confianza desde el punto de vista del género. Esto pone de manifiesto que se debe prestar atención a la diversidad tanto cultural como lingüística, en busca de una educación inclusiva y por consiguiente de una sociedad incluyente.

PALABRAS CLAVE: atención a la diversidad, marco legal, sistema educativo vasco, sensibilidad intercultural.

\section{INTERCULTURAL SENSITIVITY OF YOUNG BILINGUAL BASQUES: SOME ASPECTS TO WORK ON IN THE BASQUE EDUCATIONAL SYSTEM}

\footnotetext{
ABSTRACT: The main objective of this empirical research is to identify strengths and aspects to work on in order to attend and care for diversity in the classrooms from the point of view of intercultural awareness. To this end, after reviewing the legal framework for diversity programs, information was collected using the Intercultural Sensitivity Scale tool from 410 students (223
} 
male and 187 female) in the Autonomous Community of the Basque Country, together with descriptive information on gender, course and mother tongue(s). The results of the analysis of the data indicate that: the mother tongue is a variable to be taken into account, since it is decisive together with the gender or the course of schooling of the students. In addition, two important points to work on in the classrooms of the Basque Autonomous Community have been identified: frustration and confidence from the point of view of gender. This shows that attention must be paid to both cultural and linguistic diversity, in the search for an inclusive education and therefore an inclusive society.

KEYWORDS: Attention to diversity, legal framework, Basque educational system, intercultural sensitivity.

Recibido: 20/12/2019

Aceptado: 27/08/2020

Correspondencia: Naia Eguskiza Sánchez, Universidad del País Vasco, Facultad de Educación, Barrio Sarriena s/n, 48940 Leioa (Bizkaia). Email: naia.egusquiza@ehu.eus

\section{INTRODUCCIÓN}

En este apartado se describe el contexto de la Comunidad Autónoma Vasca desde el punto de vista del marco legal, asimismo, se define y se caracteriza el concepto que será el objeto de investigación que es la sensibilidad intercultural de los jóvenes bilingües vascos.

\subsection{El contexto vasco: marco legal}

Desde los tiempos de Heródoto (siglo $\mathrm{V}$ a.C.), quien recogió primeramente las distinciones culturales, actitudinales y credenciales entre griegos y egipcios (Adrados, 1961), hasta nuestros días, el ser humano se ha preocupado y ocupado por dichas diferencias. Esto se debe a la afluencia de gente en constante migración a escala global. Todo ello repercute en las políticas de migración, organización y configuración demográfica, socio-económica y socio-cultural, tanto de los lugares de origen como de destino, con lo que las sociedades actuales requieren de políticas que respondan a la realidad.

El sistema educativo, desde la perspectiva de pensamiento sistémico (Garciandía, 2005), ha de entenderse como un sistema complejo adaptativo (Gell-Mann, 1995). Esa adaptabilidad ha de traer consigo el paralelismo con la realidad actual que se vive en la sociedad; en el contexto al que nos referimos, en la Comunidad Autónoma del País Vasco (CAPV) ${ }^{1}$. Así pues, entendemos que la educación y sus sistemas de enseñanza-aprendizaje son la fuerza transformadora de la sociedad ya que la edu-

1. CAPV hace referencia a la Comunidad Autónoma del País Vasco, en adelante se emplearán dichas siglas. 
cación es la columna vertebral que permite avanzar a la sociedad adecuándose a las exigencias de hoy en día (Quesada, Hernández, Valenciano y Bonilla, 2019).

La sociedad vasca del siglo XXI es plurilingüe y pluricultural. Según el Observatorio Vasco de Inmigración (Ikuspegi), 76.914 personas residentes en la CAPV de entre 0 y 24 años tienen al menos un progenitor de origen extranjero. Más de la mitad (unas 46.609 personas, correspondientes al 60,6\%), son hijos e hijas de personas de origen extranjero que o bien han nacido en el Estado español o han sido reagrupadas antes de los 4 años de edad (Mendoza y Otero, 2017).

En este contexto se han elaborado programas, planes y orientaciones para el tratamiento de la diversidad en el ámbito escolar de la CAPV a lo largo de los últimos veinte años. Las primeras orientaciones para el tratamiento de la diversidad en educación primaria (Tranche, García y Rubio, 1996) ofrecieron pautas para la reflexión sobre la práctica docente, tales como que el docente debía: (1) ayudar al alumnado a ser protagonista de su propio aprendizaje, (2) ofrecer ayuda personalizada, (3) flexibilizar el programa, (4) crear un clima adecuado en el aula, (5) informar sobre el proceso de aprendizaje, (5) formar parte de un equipo docente, (6) plantear temas con un enfoque globalizador, (7) hacer disfrutar aprendiendo al alumnado, (8) adaptarse al ritmo de clase, (9) favorecer el aprendizaje cooperativo para crear lazos de ayuda, aceptación, respeto y comprensión de las diferencias, (10) potenciar las relaciones entre iguales, y (11) facilitar el aprendizaje en grupos.

En el año 2002 se especifican algunas estrategias para el tratamiento de la diversidad en Educación Secundaria Obligatoria (etapa en la que esta investigación pone el foco de atención), concretamente se busca facilitar "la incorporación e integración social y educativa de todo el alumnado, para evitar la exclusión social y cultural, desarrollando actitudes de comunicación y de respeto mutuo entre todo el alumnado independientemente de su origen cultural, lingüístico y étnico" (Departamento de Educación, Universidades e Investigación, 2002, p. 4). Para este fin, se deben potenciar los aspectos específicos de la cultura propia, y se propone la incorporación al Proyecto Educativo y Curricular de los objetivos socioculturales de carácter intercultural de compensación de las desigualdades de origen, así como de los elementos culturales propios (Departamento de Educación, Universidades e Investigación, 2002). Este mismo año, La Universidad Nacional de Educación a Distancia (UNED, 2002) publica la "Guía Inter" para aplicar la educación intercultural en la escuela (Aguado et al., 2006). Entre otros aspectos, se dan orientaciones para desafiar la perspectiva de homogeneidad, la idea de que la escuela se basa en la mera transmisión de conocimiento, la asociación de la diversidad cultural con determinadas categorías sociales y la idea de que la educación intercultural consiste únicamente en la celebración festiva de la diversidad. Asimismo, esta guía propone favorecer la perspectiva de la educación intercultural como base para entender la diversidad como una riqueza común.

En el marco del Plan Vasco de Inmigración de 9 de diciembre de 2003, se publica el programa para la atención al alumnado inmigrante (Gobierno Vasco, 2003), donde se define la CAPV como tierra de acogida de inmigrantes que principalmente provienen de Latinoamérica, del Magreb, de Europa del Este y de África Subsahariana. Se estable- 
cen una serie de principios, que entre otros son los siguientes: el principio de integración a través de la educación intercultural con el objetivo de respetar la diversidad; el principio de igualdad, para evitar actitudes discriminatorias; y el principio de interculturalidad, que fomenta el reconocimiento y la valoración de otras culturas y lenguas.

Un año después, el Departamento de Educación, Universidades e Investigación de la Administración de la CAPV especifica las orientaciones para la elaboración del plan de acogida del alumnado inmigrante, donde se define de la siguiente manera:

Un protocolo de actuaciones cuyo objetivo es facilitar la adaptación del nuevo alumnado al centro escolar. Las orientaciones que se dan se refieren a los alumnos y alumnas que provienen de otros países y/o desconocen las lenguas oficiales de la CAPV y deben llevar un proceso de adaptación escolar y aprendizaje de la lengua. (Departamento de Educación Universidades e Investigación, 2004, p. 8)

Este plan de acogida hace hincapié en la información que se debe dar a las familias. El Gobierno Vasco (2007) publica en diferentes lenguas las características y el organigrama del sistema educativo vasco, el proceso de matriculación del alumnado, el calendario escolar y la organización de un centro docente. Este mismo año, el Departamento de Educación, Universidades e Investigación presenta el programa de interculturalidad y de inclusión del alumnado recién llegado ${ }^{2}$. Dicho programa da especial importancia al bienestar emocional y afectivo del alumnado recién llegado; uno de los objetivos es potenciar la educación intercultural, basada en la igualdad, la solidaridad y el respeto a la diversidad y, por último, hacer notar que una línea de trabajo es la sensibilización de toda la comunidad educativa.

\subsection{La sensibilidad intercultural en la escuela}

La sensibilidad intercultural se define como una actitud que favorece la aceptación de las culturas ajenas:

La sensibilidad intercultural es una actitud que predispone a realizar conductas positivas ante la diversidad cultural (Ruiz-Bernardo, Ferrández-Berrueco y Sales-Ciges, 2012). Así, una mayor sensibilidad intercultural favorece la aceptación, comprensión y aprecio de las diferencias culturales (Chen y Starosta, 1996). (Micó-Cebrián, 2019, p. 41)

Chen y Starosta (2000) afirman que es necesaria una sensibilidad intercultural para una adecuada comunicación intercultural: "Successful intercultural communication demands interactants' ability of intercultural awareness by learning cultural similarities and differences, while the process of achieving awareness of cultural similarities and differences is enhanced and buffered by the ability of intercultural sensitivity" (p. 4). Los autores mencionados (2000) especifican seis elementos que componen la sensibilidad intercultural: autoestima, autocontrol, mente abierta, empatía, interacción y no-juicio (Alsina, 1999; Oghena, 2005; Aguaded, 2006; Alonso

2. El programa de interculturalidad y de inclusión del alumnado recién llegado es presentado en 2007 por el Departamento de Educación, Universidades e Investigación en una jornada celebrada en Miñano el 18/05/2007 con la participación de diversos expertos. 
et al., 2007; Desco, 2012). Tomando como base esta conceptualización crearon una escala de sensibilidad intercultural que recoge las seis características mencionadas. Tal y como se especifica en el apartado siguiente, esta escala se ha empleado para realizar la investigación cuantitativa en una muestra de 410 jóvenes bilingües vascos.

El contexto educativo tiene el objetivo de sensibilizar al alumnado para formar personas interculturalmente competentes. Las actitudes relacionadas con esta competencia incluyen aquéllas que favorecen la escucha, el contraste de opiniones y el respeto hacia los pareceres de los demás, así como el interés por la comunicación intercultural. Un elemento básico de esta competencia es la valoración positiva de la diversidad cultural y de las lenguas como medio para comunicarse y relacionarse interpersonalmente con personas de otras culturas (Micó-Cebrián, Cava y Buelga, 2019).

En cuanto a la función docente, en el actual sistema educativo contemporáneo se requieren profesores y profesoras que, entre otras características, además de su liderazgo y empoderamiento (Fernández, Ruiz, Gómez y Palacios, 2019), sean mediadores interculturales, animadores de una comunidad educativa intercultural, conductores culturales y sean capaces de crear situaciones de aprendizaje significativo, gestionar la heterogeneidad, reflexionar críticamente sobre su práctica y sobre el lugar que le corresponde a la educación en la sociedad actual (Pegalajar y Colmenero, 2017).

Gran parte de los estudios realizados sobre la medición y evaluación de la sensibilidad intercultural son provenientes de la cultura anglosajona. "Los estudios sobre sensibilidad intercultural son muy escasos, a pesar del interés en formar a niños y jóvenes como ciudadanos de un mundo cada vez más plural y diverso" (Sanhueza, 2010, p. 57). A pesar de su escasez, a continuación, exponemos algunos de los estudios realizados, estudios realizados tanto en centros de enseñanza (escuelas e institutos) como a nivel social. De Santos (2004), explica en su tesis doctoral el desarrollo de la competencia intercultural en el alumnado universitario. Un año después, en el año 2005, Vilà recoge en su tesis la sensibilidad intercultural en el alumnado del primer ciclo de la ESO. El planteamiento es evaluar la sensibilidad intercultural en la adolescencia, para posteriormente poder intervenir en el área de Educación Secundaria. Aguaded (2006), evalúa la sensibilidad intercultural del alumnado de secundaria para identificar las necesidades educativas. El trabajo de Sanhueza y Cardona (2009) estudia la sensibilidad intercultural en el alumnado de Educación Primaria en dos provincias de Alicante. Un año después, Sanhueza (2010) presenta su tesis doctoral, mostrando el estudio exploratorio sobre sensibilidad intercultural realizado en la provincia de Alicante con alumnado de Educación Primaria y Secundaria. Hernández (2011) también presenta en su tesis la competencia intercultural del alumnado de Educación Primaria, evaluando y diseñando un plan de intervención para su desarrollo. Otro de los estudios es el publicado por Vázquez et al., 2012, en la población joven de Andalucía. La tesis de RuizBernardo es del mismo año (2012b), basada en la validación de un instrumento para la medición de la sensibilidad intercultural en la provincia de Castellón.

El presente estudio se centra en la realidad de la CAPV como tierra de acogida, puesto que describe el marco legal de este territorio, además de las características en torno a la sensibilidad intercultural de los jóvenes bilingües vascos, con el objetivo de identificar fortalezas y aspectos a trabajar para atender y cuidar la diversidad de 
las aulas desde el punto de vista de la sensibilización intercultural. Así el estudio fija tres interrogantes: ¿Qué nivel de desarrollo de sensibilidad intercultural posee el alumnado joven bilingüe vasco de este estudio? ¿Qué dimensiones son las más y las menos desarrolladas (sentimientos de frustración en las relaciones con personas de otras culturas, sentimientos agradables hacia la relación e interacción, percepciones y comportamientos que manifiestan la sensibilidad intercultural, la interacción adulta y respetuosa, la autoconfianza y la atención durante la interacción)? ¿Influye en ello el género, el curso de escolarización y la lengua materna?

Para obtener respuestas a las preguntas de investigación planteadas, tal y como se explica en el siguiente apartado las variables objeto de estudio son la lengua materna, el género y el curso de escolarización, con el objetivo de observar si existen diferencias significativas según dichas variables.

\section{Método}

En este segundo apartado se procede a especificar el método llevado a cabo para la realización de la presente investigación. Se define la muestra, el instrumento con el que se han recogido los datos, el procedimiento para la elaboración de la base de datos y la definición del análisis de los mismos.

\subsection{Muestra}

Para construir la base de datos objeto de estudio se ha contado con la participación de 410 estudiantes (223 alumnas y 187 alumnos, figura 1) de Educación Secundaria Obligatoria y Bachillerato, distribuidos así en seis cursos: los cuatro primeros pertenecientes a los cursos de Enseñanza Secundaria Obligatoria (grupo uno 82 estudiantes; grupo dos 87; grupo tres 76; grupo cuatro 70) y los dos consiguientes pertenecientes al Bachillerato (grupo cinco 53; grupo seis 42). La muestra se distribuye según el curso tal y como se muestra en la figura 2.

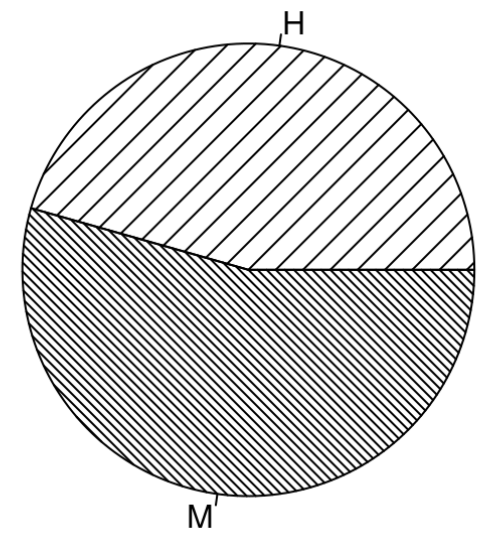

Figura 1. Distribución del alumnado por género 


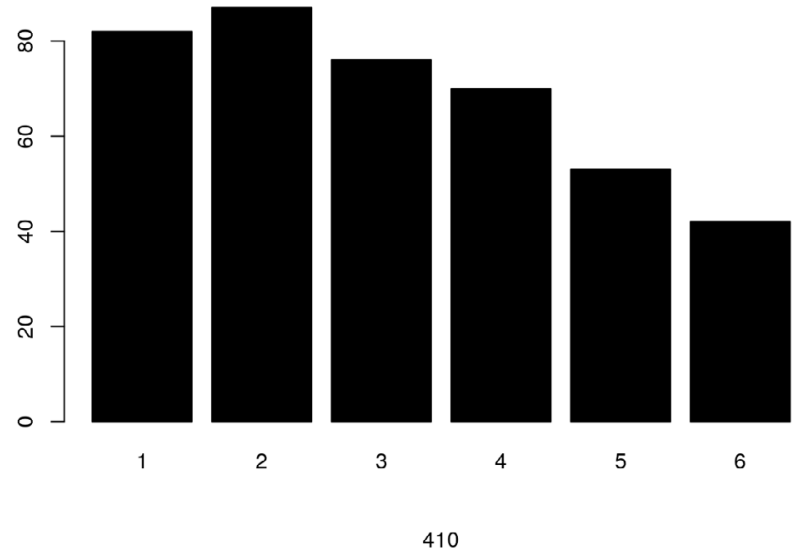

Figura 2. Distribución del alumnado por curso

299 estudiantes tienen el euskera como lengua materna, 88 el castellano y 23 otras lenguas. Estos últimos han sido etiquetados como lengua de origen extranjero. Ya que, el euskera y el castellano son las lenguas cooficiales en el territorio. En la figura 3 se muestra la distribución según la lengua materna.

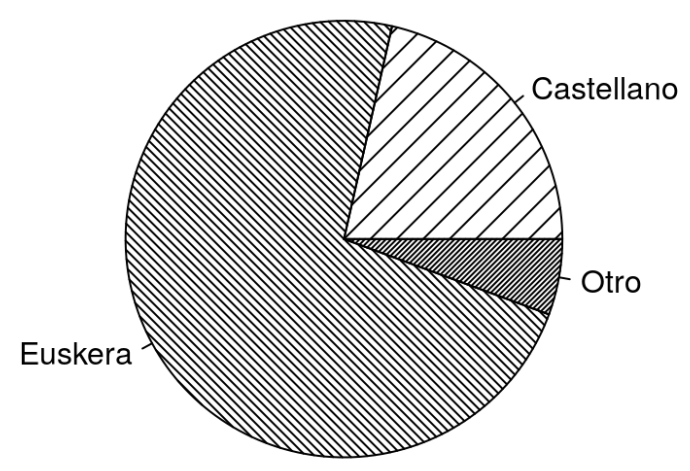

Figura 3. Distribución del alumnado por lengua materna

Las diferentes distribuciones del alumnado según las variables descriptoras son uniformes exceptuando la distribución por curso según la lengua materna del alumnado, ya que, no es uniforme, así muestra el gráfico (figura 4) de la distribución coloreado según los residuos estandarizados de ji cuadrado ( $\chi^{2}$ (g.l. 10) $=37,695 ; p$ $=0,000$ ). Resultado que representa una $V$ de Cramer $=0,21$ que indica una relación mediana con los grados de libertad obtenidos. 


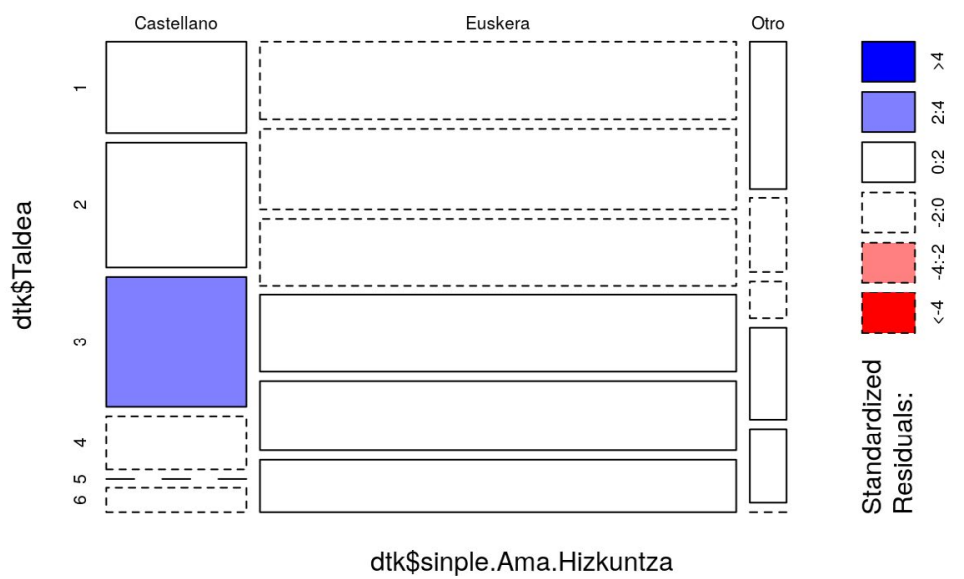

Figura 4. Relación entre descriptores: Distribución no uniforme según LM y curso

\subsection{Instrumento}

Para la recogida de datos el alumnado ha respondido un cuestionario diseñado ad hoc. La parte I contiene variables demográficas tales como el género, la lengua materna y el curso de escolarización (variables que han sido objeto de estudio); y la parte II incluye el cuestionario denominado Escala de Sensibilidad Intercultural (ISS: Intercultural Sensitivity Scale). Instrumento validado por Ruiz-Bernardo (2012a, 2012b) a partir del preludio de Chen y Starosta (2000).

El cuestionario se compone de 24 ítems en escala tipo Likert de 5 puntos (1=Totalmente en desacuerdo; $5=$ Totalmente de acuerdo), que se agrupan en competencias afectivas básicas (Ruiz-Bernardo, 2009; 2012b). Los ítems mencionados corresponden a seis dimensiones, ejes principales de la sensibilidad intercultural, tal y como se muestra en la figura 5.

(1) Dimensión 1: Sentimientos de frustración en las relaciones con personas de otras culturas, postura etnocéntrica (ítems 10,13,15, 18, 20, 22)

(2)Dimensión 2: Sentimientos agradables hacia la relación e interacción con personas de otras culturas (ítems 1, 5, 11, 14, 23)

(3) Dimensión 3: Percepciones y comportamientos que manifiestan la sensibilidad intercultural positiva (ítems $2,4,16,24)$

(4) Dimensión 4: Pautas para la interacción adulta y respetuosa (ítems 9, 12, 17, 19)

(5) Dimensión 5: Autoconfianza (ítems 3,6)

(6) Dimensión 6: Atención durante la interacción (ítems 7, 8, 21)

Figura 5. Principales dimensiones de la Sensibilidad Intercultural e ítems correspondientes 


\subsection{Procedimiento}

Para la recogida de datos se ha contado con la colaboración de un instituto público del contexto educativo vasco. En la fase de recogida de datos los escolares tras la entrega del cuestionario a cumplimentar, han sido informados del carácter anónimo, confidencial y voluntario de su participación. También se les ha explicado el objetivo del estudio. Se ha incidido en la importancia de responder todos los ítems. El alumnado ha dispuesto de treinta minutos para cumplimentar la escala.

\subsection{Análisis de los datos}

Para el análisis estadístico de los datos se ha empleado el lenguaje estadístico R (R Core Team, 2018). Previamente a la elaboración de la matriz básica, se han realizado los análisis pertinentes para la exploración de la sensibilidad intercultural del alumnado. Primeramente, se ha comprobado la normalidad de la muestra aplicando el test de normalidad de Lilliefors (adaptación del test de Kolmogorov-Smirnov). Dado que la distribución de las dimensiones no procede de una distribución normal, se han aplicado técnicas no paramétricas. Así pues, en el caso de categorías dicotómicas se ha empleado el $U$ test de Mann-Withney-Wilcoxon y, en cuanto a las categorías con más de dos niveles, se ha utilizado la prueba de Kruskal-Wallis de suma de rangos (también denominada ANOVA no paramétrica). De acuerdo con la tradición investigativa en este campo, el nivel de confianza establecido es del $95 \%$, por lo que los resultados se han considerado estadísticamente significativos cuando las diferencias han sido calculadas con probabilidad menor a $\alpha=0.05$ (valor de $\mathrm{p}<0.05$ ).

\section{Resultados}

A continuación, se muestran los resultados obtenidos en esta investigación. Las dimensiones correlacionan entre sí, según se muestra en la tabla 1. En esos datos observamos que a mayor frustración (dimensión 1) menores son los sentimientos agradables (dimensión 2), aún menor es la sensibilidad entre culturas (dimensión 3), menor el respeto en la interacción (dimensión 4), muestran menos autoconfianza (dimensión 5) y puntúan más bajo en la atención durante la comunicación (dimensión 6).

La coherencia interdimensional de la herramienta es visible en las correlaciones entre las puntuaciones de las diferentes dimensiones, calculadas mediante el coeficiente $\rho$ de correlación de Spearman. A continuación, previamente a la realización de todos los análisis pertinentes, se expondrán los resultados en los que se han encontrado diferencias estadísticamente significativas entre descriptores de la población consideradas en el estudio (género, curso, lengua materna). Los datos se han analizado atendiendo a las seis dimensiones (sub-apartado 2.2.), tal y como se ha descrito previamente (sub-apartado 2.4.). 
Tabla 1. Coeficiente de correlación de Spearman

\begin{tabular}{|l|c|c|c|c|c|c|}
\hline & $\begin{array}{c}\text { dim.1 } \\
\text { frustración }\end{array}$ & $\begin{array}{c}\text { dim.2. } \\
\text { s.agradables }\end{array}$ & $\begin{array}{c}\text { dim.3. } \\
\text { sensibilidad }\end{array}$ & $\begin{array}{c}\text { dim.4 } \\
\text { respeto }\end{array}$ & $\begin{array}{c}\text { dim.5. } \\
\text { autoconfianza }\end{array}$ & $\begin{array}{c}\text { dim.6. } \\
\text { atención }\end{array}$ \\
\hline $\begin{array}{l}\text { dim.1 } \\
\text { frustración }\end{array}$ & 1.000 & -0.369 & -0.440 & -0.245 & -0.198 & -0.490 \\
\hline $\begin{array}{l}\text { dim.2. } \\
\text { s.agradables }\end{array}$ & -0.369 & 1.000 & 0.403 & 0.444 & 0.243 & 0.494 \\
\hline $\begin{array}{l}\text { dim.3. sensibi- } \\
\text { lidad }\end{array}$ & -0.440 & 0.403 & 1.000 & 0.297 & 0.314 & 0.446 \\
\hline $\begin{array}{l}\text { dim.4. } \\
\text { respeto }\end{array}$ & -0.245 & 0.444 & 0.297 & 1.000 & 0.190 & 0.318 \\
\hline $\begin{array}{l}\text { dim.5. } \\
\text { autoconfianza }\end{array}$ & -0.198 & 0.243 & 0.314 & 0.190 & 1.000 & 0.329 \\
\hline $\begin{array}{l}\text { dim.6. } \\
\text { atención }\end{array}$ & -0.490 & 0.494 & 0.446 & 0.318 & 0.329 & 1.000 \\
\hline
\end{tabular}

La primera dimensión, la que recoge los sentimientos de frustración en las relaciones correlaciona leve y negativamente con el curso del alumnado $(\tau=-0.122$, $p$ $=0.0009054)$. En la Figura 6 se muestra la expresión gráfica de esta relación. Según avanza el curso, se encuentra levemente un menor nivel de frustración en las relaciones interculturales.

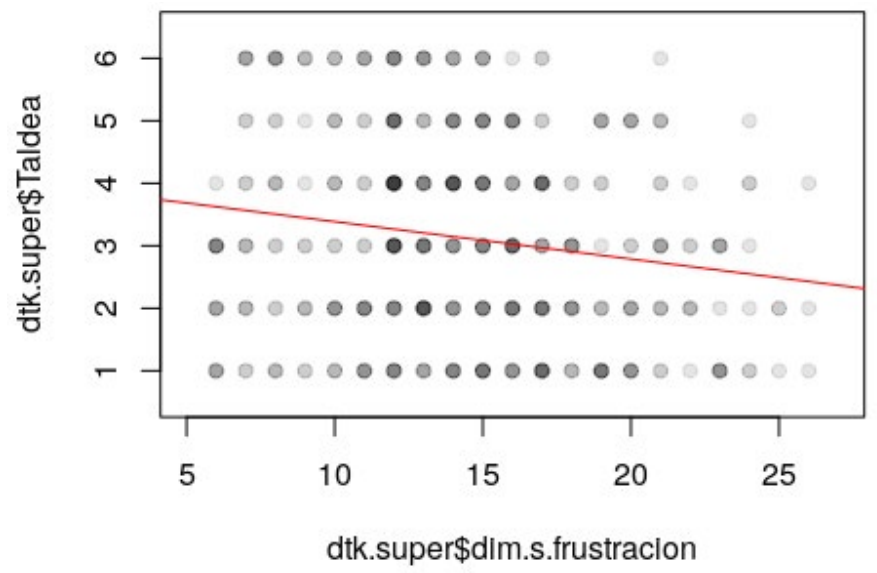

Figura 6. Dimensión 1: distribución por curso

En cuanto a la lengua materna, se han encontrado diferencias significativas en esta primera dimensión. Diferencias significativas de baja intensidad en el par castellanoeuskera, no son estadísticamente significativas las diferencias que se aprecian con respecto a otras lenguas maternas; en la figura 7 se representa la distribución de las puntuaciones según la lengua materna. 


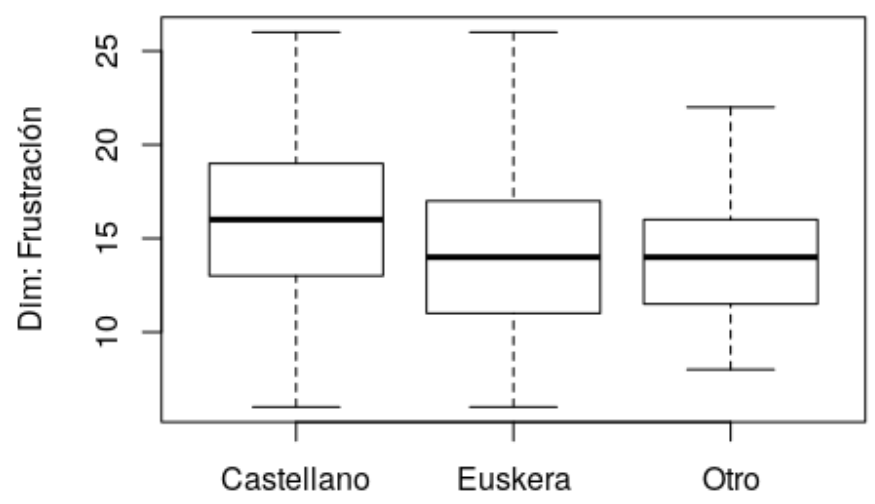

Lengua materna

Figura 7. Dimensión 1: distribución por lengua materna

En la segunda dimensión, la que conforman los sentimientos agradables hacia la relación e interacción con personas de distintas culturas a la propia, no se han encontrado diferencias estadísticamente significativas en base al género, curso, ni lengua materna, con lo cual, las puntuaciones en sentimientos agradables de todo el alumnado se distribuyen homogéneamente, en cuanto a los factores de análisis.

Las percepciones y comportamientos que manifiestan la sensibilidad intercultural positiva, los ítems pertenecientes a la tercera dimensión, muestran resultados estadísticamente significativos atendiendo a la lengua materna del alumnado. Aplicando el U test de Mann-Withney-Wilcoxon a los tres pares, detectamos que hay diferencias significativas entre las puntuaciones reportadas por el alumnado con lenguas de origen inmigrante y los hablantes de lenguas oficiales (U-test(castellano-otro) $=1833.5, p$-valor $=0.0004987$ y U-test(euskera-otro) $=523$, $p$-valor $=0.0001764)$; no encontramos diferencias significativas entre el alumnado de una u otra lengua oficial. Como observamos en la Figura 8, el alumnado más sensible interculturalmente es el de lengua materna de origen extranjero.

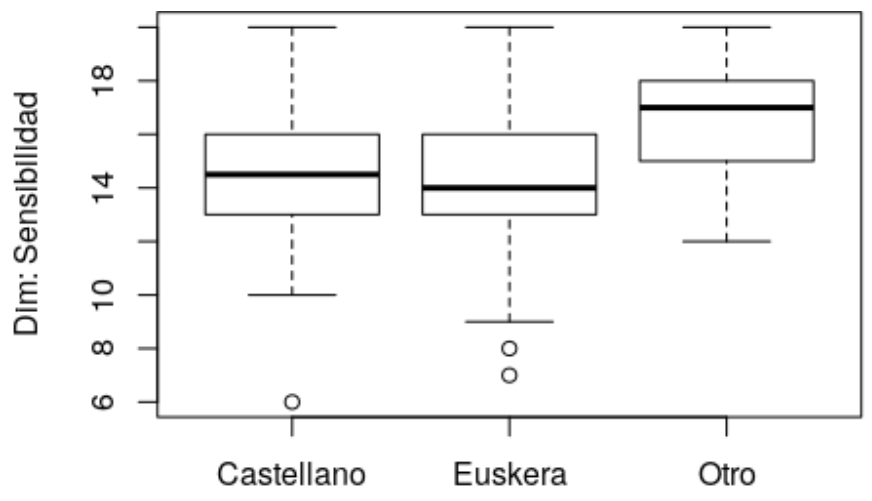

Lengua materna

Figura 8. Dimensión 3: distribución por lengua materna 
Las pautas para la interacción respetuosa, recogidas en la cuarta dimensión, no muestran diferencias estadísticamente significativas en las variables descriptoras de este estudio.

La quinta dimensión, encargada de describir la autoconfianza del alumnado en las relaciones con personas de culturas distintas a la propia, se muestran diferencias estadísticamente significativas según el género (figura 9) del alumnado y también según la lengua materna de éste (figura 10).

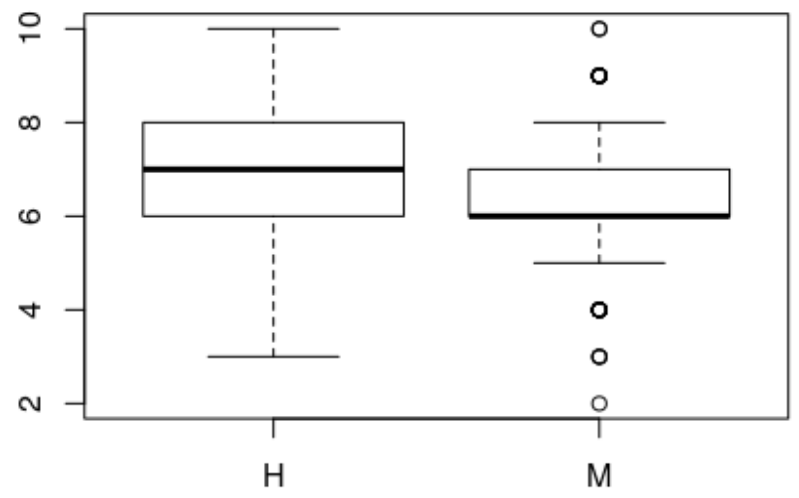

Figura 9. Dimensión 5: distribución por género

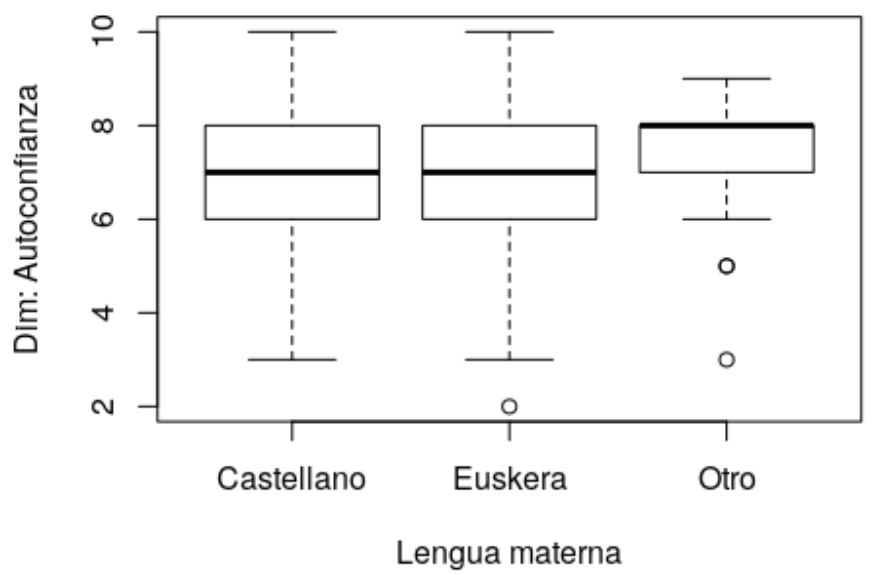

Figura 10. Dimensión 5: distribución por lengua materna

Tal y como se muestra en la figura 9, los varones reportan levemente mayor autoconfianza en las relaciones interculturales que las mujeres (U-test(femenino-masculino) $=24235$, p-valor $=0.002913)$.

La figura 10 muestra las diferencias según la lengua materna del alumnado en base a la sexta dimensión. Dichas leves diferencias son estadísticamente significati- 
vas según el test $U$ de Mann-Withney-Wilcoxon entre los grupos de lengua materna euskera y lenguas de origen inmigrante $(U=2366$, p-valor $=0.011)$.

En la sexta y última dimensión se han recogido los datos correspondientes a la atención durante la interacción del alumnado. Los datos no han mostrado significación estadística; el alumnado muestra homogeneidad en la puntuación de dicha dimensión.

\section{DisCuSión Y CONCLUSIONES}

Como se ha mostrado en el trabajo, en tres de las dimensiones se han encontrado diferencias estadísticamente significativas según alguna variable descriptora. La dimensión 1 que envuelve los sentimientos de frustración en las relaciones con personas de otras culturas; la dimensión 3, referente a las percepciones y comportamientos que manifiestan la sensibilidad intercultural positiva; y, la dimensión 5, que refiere a la autoconfianza. Las diferencias han sido estadísticamente significativas en cuanto a la lengua materna en las tres dimensiones. En la dimensión 1 ha sido el curso de escolarización y en la dimensión 5 el género. En las otras tres dimensiones no se han encontrado diferencias estadísticamente significativas, estas son: la dimensión 2, referente a los sentimientos agradables hacia la relación e interacción con personas de otras culturas; la dimensión 4, que recoge las pautas para la interacción adulta y respetuosa; y, la dimensión 6, que muestra la atención durante la interacción. En la tabla 2 se muestran resaltados los cuadros que corresponden a los datos obtenidos estadísticamente significativos contrastando las relaciones entre las seis dimensiones y las tres variables.

Tabla 2. Resumen de los datos obtenidos

\begin{tabular}{|l|c|c|c|c|c|c|}
\hline & $\begin{array}{c}\text { dim.1. } \\
\text { frustración }\end{array}$ & $\begin{array}{c}\text { dim.2. } \\
\text { s.agradables }\end{array}$ & $\begin{array}{c}\text { dim.3. } \\
\text { sensibilidad }\end{array}$ & $\begin{array}{c}\text { dim.4. } \\
\text { respeto }\end{array}$ & $\begin{array}{c}\text { dim.5. } \\
\text { autoconfianza }\end{array}$ & $\begin{array}{c}\text { dim.6. } \\
\text { atención }\end{array}$ \\
\hline Género & & & & & & \\
\hline Curso & & & & & & \\
\hline Lengua materna & & & & & & \\
\hline
\end{tabular}

Partiendo de los tres interrogantes establecidos inicialmente: el nivel de desarrollo de la sensibilidad intercultural, las dimensiones más desarrolladas y menos desarrolladas, la influencia del género, el curso de escolarización y la lengua materna los resultados obtenidos indican que, en términos generales, los sujetos poseen una competencia de sensibilidad intercultural homogénea, lo que nos lleva a afirmar que, basándonos en los resultados obtenidos a partir de nuestros datos, la lengua materna es una variable a tener en cuenta, ya que es decisiva junto con el género o el curso de escolarización del alumnado. Además, se han identificado principalmente dos puntos importantes a trabajar en las aulas de la CAPV que son la frustración y la confianza desde el punto de vista del género. 
La frustración es el estrés que surge cuando no hay una comunicación intercultural adecuada (Chen y Starosta, 2000) y puede tener las siguientes consecuencias negativas, tal y como se observa en los resultados obtenidos:

a) Menos sentimientos agradables

b) Menor sensibilidad intercultural

c) Menor respeto en la interacción

d) Menor autoconfianza

e) Menor atención durante la comunicación

Además, según los resultados obtenidos, las mujeres tienden a sentirse menos seguras a la hora de relacionarse de manera intercultural. Por lo tanto, se debe trabajar y ofrecer orientaciones, planes y programas a nivel territorial para desarrollar la sensibilidad intercultural desde el punto de vista del género, en un clima de convivencia, tolerancia y respeto a la diversidad que presenta la sociedad de hoy en día.

Todo esto pone de manifiesto la necesidad inminente de prestar atención a la diversidad tanto cultural como lingüística, en busca de una educación inclusiva y por consiguiente de una sociedad incluyente. Tal y como se ha mencionado en el marco teórico, además de programas que respalden, apoyen y abarquen la interculturalidad, el respeto y la diversidad dentro del contexto educativo, hace falta el liderazgo y empoderamiento docente para la mediación intercultural, y así se ponga en valor la interculturalidad, la heterogeneidad y la diversidad.

\section{REFERENCIAS BIBLIOGRÁFICAS}

Adrados, F. R. (1961). Introducción a Heródoto. Estudios clásicos, 6(32), 7-31.

Aguaded, E. M. (2006). La educación de competencias básicas para el desarrollo de la ciudadanía intercultural en la ESO. En Actas del V Congreso Internacional "Educación y Sociedad: Retos del s. XXI, 8 pp.

Aguado, T., Álvarez, B., Ballesteros, B., Castellano, J. L., Cuevas, L., Jaurena, I. G., ... y Hernández, C. (2006). Guía inter. Una guía práctica para aplicar la Educación Intercultural en la escuela. España: Secretaria General Técnica.

Alonso, L. M., Murcia, G., Murcia, J., Herrera, D., Gómez, D., Comas, M., .. y Ariza, P. (2007). Autoestima y relaciones interpersonales en jóvenes estudiantes de primer semestre de la División Salud de la Universidad del Norte, Barranquilla (Colombia). Revista Científica Salud Uninorte, 23(1).

Alsina, M. R. (1999). La comunicación intercultural. Barcelona: Anthropos.

Chen, G. M. y Starosta, W. J. (2000). The development and validation of the intercultural sensitivity scale. Human Communication, 3, 1-15.

De Santos, F. J. (2004). Desarrollo de la competencia intercultural en alumnado universitario: una propuesta formativa para la gestión de empresas multiculturales. [Tesis doctoral]. Barcelona: Universidad de Barcelona. 
DESCO (Centro de Estudios y Promoción del Desarrollo) (2012). Alternativas de desarrollo estrategias metodológicas para la construcción de una cultura de paz en el aula. Programa Regional Sur Unidad de Comunicación e Incidencia. Perú.

Fernández, R. G., Ruiz, A. P., Gómez, E. L. y Palacios, S. G. (2019). Explorando el liderazgo pedagógico del docente: su dimensión formativa. Contextos educativos: Revista de educación, 24, 9-25. https://doi.org/10.18172/con.3936

Garciandía, J. A. (2005). Pensar sistémico: una introducción al pensamiento sistémico. Editorial Pontificia Universidad Javeriana.

Gell-Mann, M. (1995). The Quark and the Jaguar: Adventures in the Simple and the Complex. Macmillan.

Gobierno Vasco (2003). Programa para la atención del alumnado inmigrante (en el marco del Plan Vasco de Inmigración de 9 de diciembre de 2003). http://www. euskadi.eus/contenidos/documentacion/inn_doc_esc_inclusiva/es_def/adjuntos/ diversidad/120004c_Doc_EJ_programa_alumnado_inmigrante_c.pdf

GobiernoVasco (2007). Programa de interculturalidad y de inclusión del alumnado recién llegado. http://www.euskadi.eus/contenidos/documentacion/inn_doc_esc_inclusiva/es_def/adjuntos/diversidad/120008c_Doc_EJ_Programa_Interculturalidad_c.pdf

Hernández, J. M. (2011). La competencia intercultural en alumnado de educación primaria: diseño y evaluación de un plan de intervención para su desarrollo. [Tesis doctoral]. Alicante: Universidad de Alicante, Facultad de Educación.

Micó-Cebrián, P., Cava, M. J. y Buelga, S. (2019). Sensibilidad intercultural y satisfacción con la vida en alumnado autóctono e inmigrante. Educar, 55(1), 39-57. http:// doi.org/10.5565/rev/educar.965

Oghena, Y. (2005). ¿Por qué la confianza? Revista CIDOB d'Afers Internacionals, 61$62,7-16$.

Otero, B. y Mendoza, K. (2017). La diversidad infantil y juvenil en la CAE. Las (mal) Ilamadas segundas generaciones. Bilbao: Universidad del País Vasco.

Pegalajar, M. D. C. y Colmenero, M. D. J. (2017). Actitudes y formación docente hacia la inclusión en Educación Secundaria Obligatoria. Revista electrónica de investigación educativa, 19(1), 84-97. http://doi.org/10.24320/redie.2017.19.1.765

Quesada, E. C., Hernández, C., Valenciano, E. M. y Bonilla, O. S. (2019). Desafíos de la Política Educativa para el Desarrollo Sostenible en Costa Rica. Revista latinoamericana de educación comparada, 9(14), 105-122. Recuperado de https:// dialnet.unirioja.es/descarga/articulo/6799115.pdf

R Core Team (2018). R: A language and environment for statistical computing. R. Foundation for Statistical Computing, Vienna, Austria. https:/www.R-project.org/

Ruiz-Bernardo, M. P. (2009). Estudio de la sensibilidad intercultural en la provincia de Castellón. Jornades de Foment de la Investigació. Forum de recerca, 15, 220229. http://hdl.handle.net/10234/77668. 
Ruiz-Bernardo, M. P. (2012a). Revisión lógica y empírica para la adaptación de un cuestionario de sensibilidad intercultural. Fòrum de recerca, 17, 381-402. http:// doi.org/10.6035/ForumRecerca.2012.17.24

Ruiz-Bernardo, M. P. (2012b). Validación de un instrumento para el estudio de la sensibilidad intercultural en la provincia de Castellón. [Tesis doctoral]. Universidad Jaume I de Castellón, Departamento de Educación.

Sanhueza, S. (2010). Sensibilidad Intercultural: Un estudio exploratorio con alumnado de Educación Primaria y Secundaria en la provincia de Alicante. [Tesis doctoral]. Alicante: Universidad de Alicante.

Sanhueza, S. y Cardona, C. (2009). Evaluación de la sensibilidad intercultural en alumnado de educación primaria escolarizado en aulas culturalmente diversas. Revista de Investigación Educativa, 27(1), 247-262. http://hdl.handle.net/10201/99261

Tranche, J. L., García, A. y Rubio, T. (1999). Orientaciones para el tratamiento de la diversidad en Educación Primaria. Gobierno Vasco, Instituto para el Desarrollo Curricular y la Formación del Profesorado.

Uned. (2002). Guía inter: Una guía práctica para aplicar la educación intercultural en la escuela. http://www.euskadi.eus/contenidos/documentacion/inn_doc_esc_inclusiva/es_def/adjuntos/diversidad/120003c_Pub_UE_Guia_Inter.pdf

Vázquez, O., Fernández, M. A., Mora, N. G. y Fernández, M. (2012). La sensibilidad intercultural en la población joven andaluza. Revista de Estudios de Juventud, 97, 37-51.

Vilà, R. (2005). La competencia comunicativa intercultural. Un estudio en el Primer Ciclo de la ESO. [Tesis doctoral]. Barcelona: Universidad de Barcelona, Facultad de Pedagogía, Departamento de Métodos de Investigación y Diagnóstico en Educación. 